\title{
Interplay between electronic topology and crystal symmetry: Dislocation-line modes in topological band insulators
}

\author{
Robert-Jan Slager, ${ }^{1}$ Andrej Mesaros, ${ }^{2}$ Vladimir Juričić, ${ }^{3}$ and Jan Zaanen ${ }^{1}$ \\ ${ }^{1}$ Instituut-Lorentz for Theoretical Physics, Universiteit Leiden, P.O. Box 9506, 2300 RA Leiden, The Netherlands \\ ${ }^{2}$ Department of Physics, Boston College, Chestnut Hill, Massachusetts 02467, USA \\ ${ }^{3}$ Institute for Theoretical Physics, Utrecht University, Leuvenlaan 4, 3584 CE Utrecht, The Netherlands \\ (Received 18 February 2014; revised manuscript received 21 November 2014; published 1 December 2014)
}

\begin{abstract}
We elucidate the general rule governing the response of dislocation lines in three-dimensional topological band insulators. According to this K-b-t rule, the lattice topology, represented by dislocation lines oriented in direction $\mathbf{t}$ with Burgers vector $\mathbf{b}$, combines with the electronic-band topology, characterized by the band-inversion momentum $\mathbf{K}_{\text {inv }}$, to produce gapless propagating modes when the plane orthogonal to the dislocation line features a band inversion with a nontrivial ensuing flux $\Phi=\mathbf{K}_{\text {inv }} \cdot \mathbf{b}(\bmod 2 \pi)$. Although it has already been discovered by Ran et al. [Nat. Phys. 5, 298 (2009)] that dislocation lines host propagating modes, the exact mechanism of their appearance in conjunction with the crystal symmetries of a topological state is provided by the K-b-t rule. Finally, we discuss possible experimentally consequential examples in which the modes are oblivious to the direction of propagation, such as the recently proposed topologically insulating state in electron-doped $\mathrm{BaBiO}_{3}$.
\end{abstract}

DOI: 10.1103/PhysRevB.90.241403

PACS number(s): 71.10.Pm, 72.10.Fk, 73.20.-r, 73.43.-f

Topological band insulators (TBIs) represent a new class of quantum materials that, due to the presence of time-reversal symmetry (TRS), feature an insulating bulk band gap together with metallic edge or surface states protected by a $\mathbb{Z}_{2}$ topological invariant [1-4]. This $\mathbb{Z}_{2}$ classification of TBIs is a part of the classification of free gapped fermion matter in the presence of the fundamental antiunitary time-reversal and particle-hole symmetries, the so-called tenfold way [5-7]. On the other hand, topologically insulating crystals break continuous translational and rotational symmetries down to discrete symmetries mathematically characterized by the space groups. By considering the crystal symmetries, an extra layer in this $\mathbb{Z}_{2}$ classification of TBIs has been recently uncovered [8]. This space group classification of TBIs results in the enrichment of the tenfold way with extra phases, such as crystalline (or "valley") phases [9] and translationally active phases, the latter featuring an odd number of band inversions at non- $\Gamma$ points in the Brillouin zone (BZ) $[8,10]$. Dislocations are of central interest in this endeavor, being the topological defects exclusively related to the lattice translations. In two dimensions (2D), the role of these lattice defects has been recently elucidated in TBIs [10,11], as well as in topological superconductors $[12,13]$ and interacting topological states [14-16]. In particular, it has been shown that these lattice defects in two-dimensional TBIs act as probes of distinct topological states through binding of the localized zero-energy modes [10].

Although early on it was identified that in three-dimensional TBIs dislocation lines support propagating helical modes [17], the precise role of dislocations has not been explored thoroughly [18-20]. In particular, the relation between the lattice symmetry and the electronic topology, as well as the characterization of these topological states through the response of the dislocation lines, still needs to be addressed. Dislocations in three dimensions (3D) are defects with a richer structure than their two-dimensional counterparts. They form lines $\mathbf{I}(\tau)$ (parametrized by $\tau \in[0,1]$ ), characterized by a tangent vector $\mathbf{t} \equiv d \mathbf{l} / d \tau$, with the discontinuity introduced to the crystalline order described by a Burgers vector $\mathbf{b}$. Both these vectors can only be oriented along the principal axes of the crystal, and a screw (edge) dislocation is obtained when $\mathbf{b} \| \mathbf{t}(\mathbf{b} \perp \mathbf{t})$ (see Fig. 1). Moreover, the Burgers vectors are additive, and in full generality we can thus consider only dislocations with the Burgers vectors equal to Bravais lattice vectors. The crucial observation is that translational lattice symmetry is preserved along the dislocation line. Therefore, the full lattice Hamiltonian in the presence of a dislocation oriented along the $z$ axis $\left(\mathbf{t}=\mathbf{e}_{z}\right)$ can be written as

$$
H_{3 \mathrm{D}}(x, y, z)=\sum_{k_{z}} e^{i k_{z} z} H_{\mathrm{eff}}^{2 \mathrm{D}}\left(x, y, k_{z}\right) .
$$

Notice that the above 2D lattice Hamiltonian possesses the (wallpaper group) symmetry of the crystallographic plane orthogonal to the dislocation line, because the Burgers vector is a Bravais lattice vector.

On the other hand, the electronic topology of a TBI is characterized by band inversions at time-reversal invariant (TRI) momenta $\mathbf{K}_{\text {inv }}$ in the BZ [8]. A dislocation disturbs the crystalline order only microscopically close to its core. We can therefore use elastic continuum theory to describe its effect at low energies. The elastic deformation of the continuous medium is encoded by a distortion field $\boldsymbol{\varepsilon}_{i}$ of the global Cartesian reference frame $\mathbf{e}_{i}, e_{i}^{\alpha}=\delta_{i}^{\alpha}$, with $i, \alpha=$ $1,2,3$ [21,22]. The momentum near the transition from a topologically trivial to a nontrivial phase with the band gap closing at the momentum $\mathbf{K}_{\text {inv }}$ is $k_{i}=\left(\mathbf{e}_{i}+\boldsymbol{\varepsilon}_{i}\right) \cdot\left(\mathbf{K}_{\mathrm{inv}}-\mathbf{q}\right)$, with $q \ll K_{\mathrm{inv}} \sim 1 / a$ as the momentum of the low-energy electronic excitations, $\varepsilon \sim a / r, a$ is the lattice constant, and $r$ is the distance from the defect core. Therefore, the dislocation gives rise to a $U(1)$ gauge field $A_{i}=-\boldsymbol{\varepsilon}_{i} \cdot \mathbf{K}_{\mathrm{inv}}$ that minimally couples to the electronic excitations, $\mathbf{q} \rightarrow \mathbf{q}+\mathbf{A}$. The translational symmetry then implies that the gauge field has nontrivial components only in the plane orthogonal to the dislocation line, consistent with Eq. (1), carrying an effective flux $\Phi=\mathbf{K}_{\text {inv }} \cdot \mathbf{b}$, as we demonstrate below. 

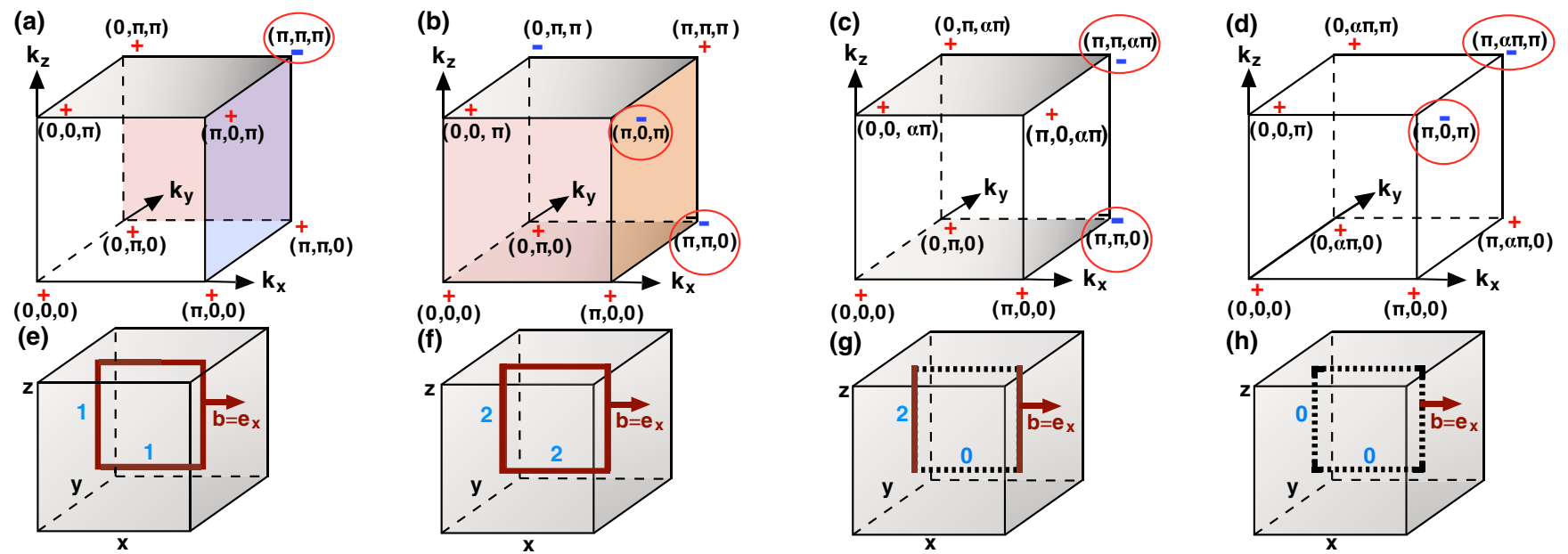

FIG. 1. (Color online) Illustration of the K-b-t rule relating the electronic topology in the momentum space, and the effect of dislocations in real space. (a)-(d) show the electronic-band topology of the $R\left[T-p 3(4)_{R}\right]$ and $M\left[T-p 3(4)_{M}\right]$ phases on a cubic lattice and the $M-R\left(p 4_{M, R}\right)$ and $X^{\prime}-R\left(p 4_{X^{\prime}, R}\right)$ weak phases on tetragonal lattices. A dislocation with $\mathbf{b}=\mathbf{e}_{x}$ acts on the encircled TRI momenta in the planes orthogonal to the dislocation line. As a result, the colored planes host a $\pi$ flux. The resulting number of Kramers pairs of helical modes along the edge and screw parts of the loop is indicated with a number (blue). (a) The symmetric $R$ phase has a topologically nontrivial plane hosting a $\pi$ flux orthogonal to any of the three crystallographic directions and hence any dislocation loop binds modes along the entire core, as shown for a loop in the $\hat{x}-\hat{z}$ plane (e). (b) In the $M$ phase, translationally active phases in the TRI planes orthogonal to $k_{z}$ and a crystalline phase in $k_{x}=\pi$ plane host $\pi$ fluxes. Hence the dislocation loop binds two pairs of modes, symmetry-protected against mixing (f). (c) In the $M$ - $R$ phase, only the TRI planes normal to $k_{z}$ host a $\pi$ flux and hence the same dislocation loop binds modes only to the edge-dislocation parts (g). These modes are not protected against mixing. (d) In the $X^{\prime}-R$ phase all TRI planes orthogonal to the dislocations lines have a trivial flux, and, according to the K-b-t rule, neither the edge nor the screw dislocation of the loop binds modes (h).

Consider an edge and a screw dislocation both oriented along the $z$ axis. We use that for the edge dislocation with Burgers vector $\mathbf{b}=a \mathbf{e}_{x}$, the dual basis in the tangent space at the point $\mathbf{r}$ is $\mathbf{E}^{x}=\left(1-\frac{a y}{2 \pi r^{2}}\right) \mathbf{e}^{x}+\frac{a x}{2 \pi r^{2}} \mathbf{e}^{y}, \mathbf{E}^{y}=\mathbf{e}^{y}, \mathbf{E}^{z}=\mathbf{e}^{z}$, while for the screw dislocation with $\mathbf{b}=a \mathbf{e}_{z}$, we have $\mathbf{E}^{x}=$ $\mathbf{e}^{x}, \mathbf{E}^{y}=\mathbf{e}^{y}, \mathbf{E}^{z}=\frac{b}{2 \pi r^{2}}\left(-y \mathbf{e}^{x}+x \mathbf{e}^{y}\right)+\mathbf{e}^{z} ; r^{2} \equiv x^{2}+y^{2}[21]$. The corresponding distortion for both an edge and a screw dislocation is then readily obtained to leading order in $a / r$ to be $\boldsymbol{\varepsilon}_{x}=\frac{y}{2 \pi r^{2}} \mathbf{b}, \boldsymbol{\varepsilon}_{y}=-\frac{x}{2 \pi r^{2}} \mathbf{b}$ (see the Supplemental Material [23] for details), and the corresponding gauge potential $\mathbf{A}(\mathbf{r})$ has nontrivial components in the plane orthogonal to the dislocation line, $\mathbf{A} \cdot \mathbf{t}=0$, and

$$
\mathbf{A}=\frac{-y \mathbf{e}_{x}+x \mathbf{e}_{y}}{2 \pi r^{2}}\left(\mathbf{K}_{\mathrm{inv}} \cdot \mathbf{b}\right) \equiv \frac{-y \mathbf{e}_{x}+x \mathbf{e}_{y}}{2 \pi r^{2}} \Phi .
$$

When this flux $\Phi(\bmod 2 \pi)$ is nonzero, the dislocations carry propagating helical modes provided that the $2 \mathrm{D}$ Hamiltonian in a TRI plane orthogonal to the dislocation line, $\hat{\mathbf{t}} \equiv \mathbf{t} /|\mathbf{t}|$, hosts a band inversion. The lattice symmetry that relates the band-inversion momenta then protects these modes. This is what we refer to as the K-b-t rule. This rule and the following descendant construction together imply that the bound states for a given $\mathbf{k} \cdot \hat{\mathbf{t}}$ momentum combine into the spectrum of the propagating helical modes along the dislocation line. For $\mathbf{k} \cdot \hat{\mathbf{t}}=\mathbf{K}_{\text {inv }} \cdot \hat{\mathbf{t}}$, the system develops a Kramers pair of true zero modes $\Psi_{0} \equiv\left(\psi_{0}, T \psi_{0}\right)^{\top}$, with $T$ as the time-reversal operator, $T^{2}=-1[10]$. This is a consequence of the fact that by definition the $\mathbf{K}_{\text {inv }}$ point is hosting a band inversion. Henceforth, the physics is essentially captured by a Dirac Hamiltonian with negative mass. Deviating from this "parent" momentum by $\mathbf{q} \cdot \hat{\mathbf{t}}$, the effective low-energy Hamiltonian for the propagating modes then generally develops a linear gap $H_{\text {eff }} \sim \mathbf{q} \cdot \hat{\mathbf{t}}$, to lowest order. The gapped Kramers pair of descendant states is then present as long as $H_{\text {eff }}(\mathbf{q})$ remains topologically nontrivial, and may then be captured by $H_{\text {eff }}=$ $v_{t} \Sigma_{3}(\mathbf{q} \cdot \hat{\mathbf{t}})+O\left(q^{2}\right)$. Here, the Pauli matrix $\Sigma_{3}$ acts in the two-dimensional Hilbert space of the dislocation modes, which are of the form $\Psi_{q_{t}} \equiv\left[\psi_{0} e^{i(\mathbf{q} \cdot \hat{\mathbf{t}})(\mathbf{r} \cdot \hat{\mathbf{t}})},\left(T \psi_{0}\right) e^{-i(\mathbf{q} \cdot \hat{\mathbf{t}})(\mathbf{r} \cdot \hat{\mathbf{t}})}\right]^{\top}$, and $v_{t}$ is the characteristic velocity, set by the symmetries and details of the band structure.

We thus see how the dislocation line $\mathbf{t}$, the Burgers vector $\mathbf{b}$, and the TRI momenta $\mathbf{K}_{\text {inv }}$, through the $\mathbf{K}-\mathbf{b}-\mathbf{t}$ rule, combine into a precise condition determining the existence of the dislocation propagating modes in certain directions in a topologically insulating phase, consistent with the space group classification [8] (Fig. 1). By varying $\mathbf{t}$ and $\mathbf{b}$ in all directions, the number of "parent" zero modes in any projection plane is in one-to-one correspondence with the space group classification in terms of the $\mathbf{K}_{\text {inv }}$ momenta. Specifically, for a (translationally active) phase with a single $\mathbf{K}_{\text {inv }}$ momentum, edge and screw dislocations bind a single Kramers pair of helical modes if $\mathbf{K}_{\text {inv }} \cdot \mathbf{b} \neq 0$. In case of a (translationally active or a crystalline) phase with multiple $\mathbf{K}_{\text {inv }}$ momenta, a projected 2D system may also result in a double pair of modes if the effective system entails a $2 \mathrm{D}$ crystalline phase. The two pairs are then protected by the symmetries relating the $\mathbf{K}_{\text {inv }}$ momenta. Most interestingly, there is the possibility that both edge and screw dislocations bind modes in any crystal direction, resulting in propagating modes along the full dislocation loops. In particular, in a completely isotropic lattice with $O_{h}$ crystal symmetry, a strong variant of this effect can be realized: the gapless states in the dislocation loop that 
(a)

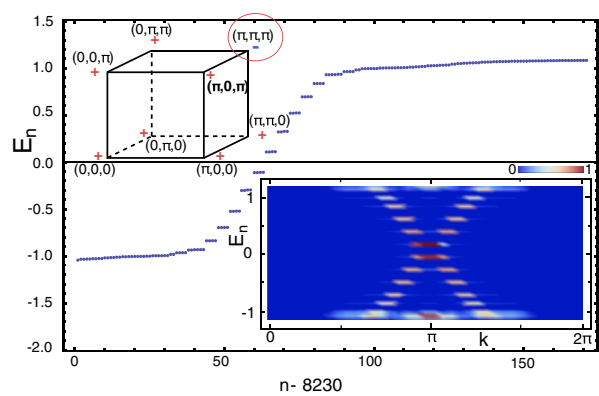

(b) $M / B=10, z=0, R$ phase

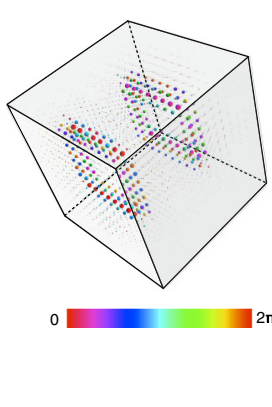

(c)

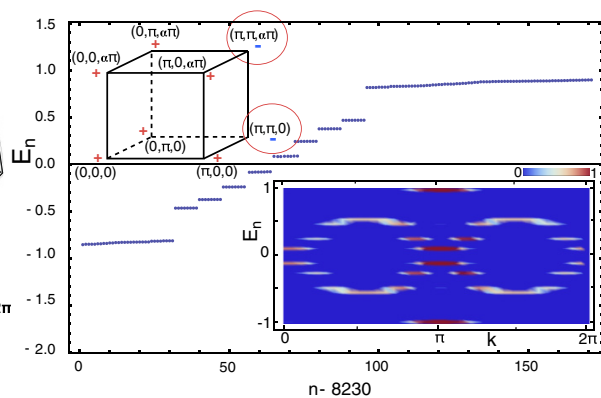

(d) $M / B=7, z=0, \alpha=0.5, M-R$ phase

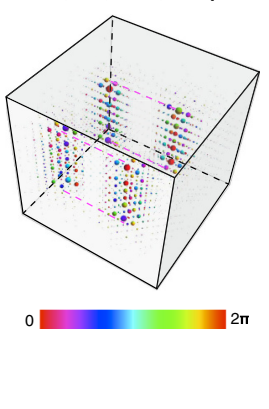

FIG. 2. (Color online) The effect of dislocation lines in 3D TBIs. Edge and screw dislocations with $\mathbf{b}=\mathbf{e}_{x}$ are treated simultaneously by considering dislocation loops of $8 \times 8$ sites within the tight-binding model (3) on the lattice with $16 \times 16 \times 16$ sites and periodic boundary conditions [23]. (a) and (c) show the spectrum of the dislocation modes traversing the gap in case of the cubic $R$ phase $\left[T-p 3(4)_{R}\right]$ and tetragonal $M-R\left(p 4_{M, R}\right)$ phases, with the corresponding electronic topological configurations shown in the insets on the upper left. The circles indicate the TRI momenta hosting band inversions that yield effective $\pi$ fluxes. Additionally, the spectral density as function of the momentum $\mathbf{k}$ along the dislocation line is displayed in the insets on the lower right. We find a single Dirac cone for the $R$ phase, and a double cone for the $M$ - $R$ phase, consistent with the K-b-t rule. The energy levels are now eightfold degenerate as both $k_{z}=0$ and $k_{z}=\pi$ planes are topologically nontrivial, and thus each yields propagating modes due to the $\pi$ flux introduced by the dislocations. The zero modes are offset due to a finite system size (see also Fig. S11 in Ref. [23]). (b) and (d) display the real space localization of the modes, where the weight of the wave function is indicated by the size of the circles and the color coding indicates the corresponding phase. Most importantly, the cubic $R$ phase features topologically protected dislocation modes propagating along the complete loop. In contrast, the $M-R$ phase binds modes only along the edge-dislocation parts. In particular, we find eight energy levels in correspondence with the number of allowed momenta along the dislocation.

propagate in a way completely oblivious to the lattice directions. In contrast, in a weak phase characterized by the weak topological index $\mathbf{M}$, protected helical modes are obtained only when the product $\mathbf{M} \cdot \mathbf{b}(\bmod 2 \pi)$ is nontrivial [3,17-19]. This is consistent with the fact that double pairs of modes originating from two $\mathbf{K}_{\text {inv }}$ not related by symmetry may be gapped out. However, according to the $\mathbf{K}-\mathbf{b}-\mathbf{t}$ rule, if the $\mathbf{K}_{\text {inv }}$ are related by symmetry, such pairs are protected from gapping out. We expect that the stability of the dislocation modes in 3D TBIs against TRI disorder is in one-to-one correspondence with the stability of the $2 \mathrm{D}$ topological phase yielding the helical modes. Therefore, the most stable dislocation modes should originate from a $2 \mathrm{D} \Gamma$ phase, followed by the ones arising from translationally active and crystalline phases [8].

These general statements can be illustrated by a simple tight-binding model with two orbitals with different parity and two spin states [24] on the cubic lattice (space group pm $\overline{3} m$ ),

$$
H_{n n}=A\left(\gamma_{1} \sin k_{x}+\gamma_{2} \sin k_{y}+\gamma_{3} \sin k_{z}\right)+M_{n n} \gamma_{0},
$$

with $\mathbf{k}$ as the electron momentum, the $\gamma$ matrices acting in the orbital and the spin spaces, and we use natural units ( $\hbar=c=a=1$ ). Topological phases of the model (3) can be tuned with the mass term $M_{n n}=m-2 B\left(3-\cos k_{x}-\right.$ $\left.\cos k_{y}-\cos k_{z}\right)$. The parameters $A(B)$ are related to the interorbital (intraorbital) hoppings, and $m$ is related to the difference in the on-site energies for different orbitals [23]. Let us assume that the system is in an $R$ phase [labeled $T-p 3(4)_{R}$ in Ref. [8]], characterized by a band inversion at the $R$ point [momentum $(\pi, \pi, \pi)$ ] in the BZ [Figs. 1(a) and 2]. If $\mathbf{b}=\mathbf{e}_{x}$ and $\mathbf{t}=\mathbf{e}_{z}$, the effective Hamiltonian in the $k_{z}=\pi$ plane reduces to the $\pi$-flux problem in the two-dimensional $M$ phase (labeled $T-p 4_{M}$ in Ref. [8]), which is defined by the band inversion at the $M$ point, and hence possesses zero modes. For the family of Hamiltonians (1) then any momentum $k_{z}=\pi+q_{z}$ infinitesimally close to $k_{z}=\pi$ yields the potential zero modes in the absence of the term $\sim \gamma_{3}$. When included, this term gives rise to the linearly dispersing propagating modes along the dislocation line, according to the above K-b-t rule. Furthermore, the same rule implies that an edge or a screw dislocation along any Bravais lattice vector in the simple cubic lattice effectively acts as a $\pi$ flux. Hence, when these defects are joined in a loop in the $x-z$ plane with $\mathbf{b}=\mathbf{e}_{x}$, we expect propagating modes, which are indeed found in our numerical computations. Clearly, TRS, together with the crystal symmetry, protects the modes from backscattering.

Next we consider the system in the three-dimensional $M$ phase $\left[T-p 3(4)_{M}\right]$, characterized by band inversions located at the momenta $M \equiv(\pi, \pi, 0), X^{\prime} \equiv(\pi, 0, \pi), Y^{\prime} \equiv(0, \pi, \pi)$, which are related by threefold rotational symmetry [Fig. 1(b) and Figs. S5 and S10 in the Supplemental Material [23]). According to the K-b-t rule, for the edge segment of the dislocation loop, $k_{z}=0$ and $k_{z}=\pi$ planes both host a $\pi$ flux, originating from the $M$ and $X^{\prime}$ points. Additionally, the $k_{x}=\pi$ plane hosts a two-dimensional crystalline phase, and thus the screw-dislocation parts also host two pairs of modes. Therefore, there is a total of two Kramers pairs of gapless dislocation modes along the loop, which are protected by symmetry. Note that their existence crucially depends on the fact that the $M$ and $X^{\prime}$ momenta are symmetry related. Were this not the case, the weak index of this $T-p 3(4)_{M}$ phase, $M_{i}=(0,0,0)$, would predict no dislocation modes at all.

In contrast, let us now break the cubic symmetry by considering the $M-R$ phase $\left(p 4_{M, R}\right)$ on a tetragonal lattice with $a_{x}=a_{y}=a, a_{z}=a / \alpha$, where $a_{i}$ is the lattice constant in the direction $\mathbf{e}_{i}$, and $\alpha \neq 1$ is the lattice deformation parameter [Figs. 1(c) and 2]. Notice here also a subtle difference between such a weak phase and a crystalline phase, which has an even number of band inversions protected by a 3D space group symmetry. The usual strong and weak indices $[3,4,17]$ in this phase are $\left(v ; M_{i}\right)=(0 ; 0,0,1)$, and thus $\mathbf{M} \cdot \mathbf{b}=0$. The $k_{z}=0$ 
and $k_{z}=\alpha \pi$ planes are topologically nontrivial (Fig. 1 and Ref. [23]). As a result, for a dislocation loop with $\mathbf{b}=\mathbf{e}_{x}$, according to the K-b-t rule, we find modes bound only to the edge-dislocation parts. As both these planes contribute the midgap states, we expect a double Kramers pair of the propagating states, which our numerical computations indeed confirm. However, these modes can mix in the dislocation loop, since no symmetry relates the momenta $M$ and $R$ yielding them.

Finally, we consider the tetragonal $X^{\prime}-R\left(Y^{\prime}-R\right)$ phase obtained by deforming the cubic lattice in the $\mathbf{e}_{y}\left(\mathbf{e}_{x}\right)$ direction with the corresponding band inversions at $(\pi, 0, \pi)[(0, \pi, \pi)]$ and $(\pi, \alpha \pi, \pi)[(\alpha \pi, \pi, \pi)]$ momenta [Fig. 1(d)]. No dislocation modes appear in the $X^{\prime}-R$ phase, whereas in the $Y^{\prime}-R$ phase only the band inversion at momentum $(\alpha \pi, \pi, \pi)$ contributes a $\pi$ flux, thus yielding the modes for both types of dislocations. These results are therefore similar as in the $R$ phase on the cubic lattice, as also confirmed by numerical computations. However, the difference is that not all directions are equivalent here, and hence the velocities of the modes along the loop are anisotropic.

The response of dislocations in TBIs is experimentally consequential as these defects are ubiquitous in any crystal. The electron-doped mixed-valent perovskite oxide $\mathrm{BaBiO}_{3}$ with a simple cubic crystal symmetry has been recently predicted to be a TBI [25]. More interestingly, the topological phase is believed to be the $R$ phase $\left[T-p 3(4)_{R}\right]$, which should host propagating gapless modes along any dislocation loop, as we have found here. The effective tight-binding model (3) describes this phase with the parameters $A=2.5 \mathrm{eV} \AA$, $B=9.0 \mathrm{eV} \AA^{2}, M=5.08 \mathrm{eV}$, and the lattice constant $a=4.35 \AA$ [25], implying that the velocity of the dislocation modes is $v=A / \hbar=4.3 \times 10^{5} \mathrm{~m} / \mathrm{s}$, while the localization length $\lambda \sim \sqrt{B /\left(12 B a^{-2}-M\right)} \simeq 4.8 \AA$ [26]. The enhanced density of states near the dislocation line in a non- $\Gamma$ TBI [27-30] should be observable by local probes, such as scanning tunneling microscopy, where the dislocation reaches the crystal surface. The surface states hybridize with the dislocation modes, and the precise local redistribution of the increased density of states can be modeled for the specific experimental situation using our theory. Angle-resolved photoemission spectroscopy may also be useful for mapping out these states, since the surface irregularities should not affect this probe.

We hope that our findings will motivate further studies of the role of dislocations in TBIs with different crystal symmetries, and especially their knotting, which should play the role of braiding for these topological defects and might be relevant for quantum computation. Finally, our results based on the "intuitive" classification of the dislocations through the Burgers vectors ultimately call for a mathematically precise characterization of these defects in terms of the homotopy classes for the discrete space groups.

Note added in proof. Recently, we became aware of the work by Shiozaki and Sato [31], where the response of dislocations in 3D TBIs was studied using $K$ theory.

This work is part of the D-ITP consortium, a program of the Netherlands Organisation for Scientific Research (NWO) that is funded by the Dutch Ministry of Education, Culture and Science (OCW). This work is supported by the Dutch Foundation for Fundamental Research on Matter (FOM). V.J. acknowledges the support of NWO. The authors acknowledge fruitful discussions with Xiao-Liang Qi and Zhi-Xun Shen.
[1] C. L. Kane and E. J. Mele, Phys. Rev. Lett. 95, 146802 (2005).

[2] L. Fu and C. L. Kane, Phys. Rev. B 74, 195312 (2006).

[3] J. E. Moore and L. Balents, Phys. Rev. B 75, 121306 (2007).

[4] L. Fu, C. L. Kane, and E. J. Mele, Phys. Rev. Lett. 98, 106803 (2007).

[5] A. P. Schnyder, S. Ryu, A. Furusaki, and A. W. W. Ludwig, Phys. Rev. B 78, 195125 (2008).

[6] S. Ryu, A. P. Schnyder, A. Furusaki, and A. W. W. Ludwig, New J. Phys. 12, 065010 (2010).

[7] A. Y. Kitaev, AIP Conf. Proc. 1134, 22 (2009).

[8] R.-J. Slager, A. Mesaros, V. Juričić, and J. Zaanen, Nat. Phys. 9, 98 (2013).

[9] L. Fu, Phys. Rev. Lett. 106, 106802 (2011).

[10] V. Juričić, A. Mesaros, R.-J. Slager, and J. Zaanen, Phys. Rev. Lett. 108, 106403 (2012).

[11] F. de Juan, A. Rüegg, and D.-H. Lee, Phys. Rev. B 89, 161117(R) (2014).

[12] D. Asahi and N. Nagaosa, Phys. Rev. B 86, 100504(R) (2012).

[13] T. L. Hughes, H. Yao, and X.-L. Qi, arXiv:1303.1539.

[14] M. Barkeshli and X.-L. Qi, Phys. Rev. X 2, 031013 (2012).

[15] M. Barkeshli, C.-M. Jian, and X.-L. Qi, Phys. Rev. B 87, 045130 (2013).
[16] A. Mesaros, Y.-B. Kim, and Y. Ran, Phys. Rev. B 88, 035141 (2013).

[17] Y. Ran, Y. Zhang, and A. Vishwanath, Nat. Phys. 5, 298 (2009).

[18] J. C. Y. Teo and C. L. Kane, Phys. Rev. B 82, 115120 (2010).

[19] Y. Ran, arXiv:1006.5454.

[20] K.-I. Imura, Y. Takane, and A. Tanaka, Phys. Rev. B 84, 035443 (2011).

[21] H. Kleinert, Gauge Fields in Condensed Matter (World Scientific, Singapore, 1989), Vol. II.

[22] R. Bausch, R. Schmitz, and L. A. Turski, Phys. Rev. Lett. 80, 2257 (1998).

[23] See Supplemental Material at http://link.aps.org/supplemental/ 10.1103/PhysRevB.90.241403 for the details of the model in Eq. (3), as well as for the technical details of the analytical and numerical description of the dislocation modes.

[24] H. Zhang, C.-X. Liu, X.-L. Qi, X. Dai, Z. Fang, and S.-C. Zhang, Nat. Phys. 5, 438 (2009).

[25] B. Yan, M. Jansen, and C. Felser, Nat. Phys. 9, 709 (2013).

[26] A. Mesaros, R.-J. Slager, J. Zaanen, and V. Juričić, Nucl. Phys. B 867, 977 (2013). 
[27] D. Hsieh, D. Qian, L. Wray, Y. Xia, Y. S. Hor, R. J. Cava, and M. Z. Hasan, Nature (London) 452, 970 (2008).

[28] Y. Tanaka, Z. Ren, T. Sato, K. Nakayama, S. Souma, T. Takahashi, K. Segawa, and Y. Ando, Nat. Phys. 8, 800 (2012).

[29] S.-Y. Xu, C. Liu, N. Alidoust, M. Neupane, D. Qian, I. Belopolski, J. D. Denlinger, Y. J. Wang, H. Lin, L. A. Wray, G. Landolt, B. Slomski, J. H. Dil, A. Marcinkova, E. Morosan,
Q. Gibson, R. Sankar, F. C. Chou, R. J. Cava, A. Bansil, and M. Z. Hasan, Nat. Commun. 3, 1192 (2012).

[30] P. Dziawa, B. J. Kowalski, K. Dybko, R. Buczko, A. Szczerbakow, M. Szot, E. Lusakowska, T. Balasubramanian, B. M. Wojek, M. H. Berntsen, O. Tjernberg, and T. Story, Nat. Mater. 11, 1023 (2012).

[31] K. Shiozaki and M. Sato, Phys. Rev. B 90, 165114 (2014). 